\title{
Direct growth of vertically aligned carbon nanotubes on a planar carbon substrate by thermal chemical vapour deposition
}

\author{
Xianming Liu ${ }^{a}$, Keith H. R. Baronian ${ }^{b}$, Alison J. Downard ${ }^{a^{*}}$ \\ ${ }^{a}$ MacDiarmid Institute for Advanced Materials and Nanotechnology, Department of \\ Chemistry, University of Canterbury, Private Bag 4800, Christchurch, New Zealand \\ ${ }^{b}$ Christchurch Polytechnic Institute of Technology, PO Box 540, Christchurch, New \\ Zealand
}

\begin{abstract}
Uniform, vertically aligned multi-walled carbon nanotube arrays (VACNTs) were grown on glassy carbon-like thin films by thermal chemical vapour deposition (CVD). Thin $(5 \mathrm{~nm})$ aluminum and iron catalyst layers were pre-deposited by evaporation on the carbon substrates and VACNTs were grown at $750{ }^{\circ} \mathrm{C}$ by water-assisted CVD using ethylene as the carbon source. The aluminum layer was shown to be essential for aligned nanotube growth. VACNT arrays adhered strongly to the carbon film with low contact resistance between the VACNTs and the substrate. The VACNT arrays grown directly on the planar conducting carbon substrate have attractive properties for use as electrodes. Excellent voltammetric characteristics are demonstrated after insulating the arrays with a dielectric material.
\end{abstract}

\footnotetext{
${ }^{*}$ Corresponding author: Fax: 64-3-3642110.

E-mail address: alison.downard@canterbury.ac.nz (A.J. Downard)
} 


\section{Introduction}

Supported arrays of vertically aligned carbon nanotubes (VACNTs) have potential applications in electronic, optoelectronic and sensing devices [1-3]. For example, VACNTs are increasingly used in bioelectrochemistry where they can exhibit fast electron transfer to solution-based redox-active species, or can act as highly conducting nanowires to connect redox enzymes to macrosized electrodes [4-9]. For applications such as these, the VACNT array must be in secure contact with a conducting support, and there must be low contact resistance between the array and the support. Clearly the simplest approach to such a format is to prepare the VACNT array directly on a conducting substrate.

Until recently, there were few examples of the growth of VACNTs on conducting substrates by the widely available thermal chemical vapour deposition (CVD) method. Some examples of VACNT growth on specific metallic substrates are the work of Gao and co-workers who reported the aligned growth of CNTs on titanium substrates with simultaneous filling of the tubes with single crystals of titanium carbide [10], and the work of Ramanath and co-workers who prepared VACNTs on indium tin oxide films on silicon [11]. More general methods were developed in the work of Liu, Zhu and co-workers where VACNT arrays were grown on nickel, titanium and tantalum sheets, and on aluminum patterned on silicon [12], while Hata and co-workers demonstrated water-assisted CVD growth of VACNTs on a number of metal foils made of nickel-based alloys with chromium and iron [13]. Most recently, some widely applicable approaches to VACNT growth on conducting substrates have been reported. Talapatra's group demonstrated growth of aligned CNTs on the metal alloy, Iconel, which can also be coated onto other metallic 
substrates, opening the possibility of VACNT growth on a variety of bulk metals [14]. On the other hand, Zachariah and co-workers showed that use of an iron/alumina composite catalyst allows successful preparation of VACNT arrays on a range of metals, alloys and ceramics [15].

To our knowledge, there have been no reports of the preparation of VACNT arrays on planar graphitic carbon substrates by thermal CVD. Aligned growth of CNTs has been reported on graphitic substrates via plasma-enhanced CVD, whereas only non-aligned growth has been reported using thermal CVD [16-18]. Graphitic carbons, in general, are inexpensive, very easy to handle and convenient materials, particularly for use as electrodes. As mentioned above, VACNTs appear to have particular advantages for some sensing applications and hence arrays directly grown on graphitic carbons are an attractive target.

We describe here the fabrication of VACNT arrays on pyrolyzed photoresist film (PPF) [19] using a straightforward water-assisted thermal CVD method. PPF is readily prepared in the laboratory as a thin film on silicon, and has similar properties to glassy carbon but with a considerably lower surface roughness [19]. A preliminary investigation of the electrochemical properties of the VACNT arrays is also reported.

\section{Experimental}

\subsection{Synthesis of VACNTs and VACNT electrodes}

Conducting carbon film (pyrolyzed photoresist film, PPF), with thickness of 1-2 $\mu \mathrm{m}$, was prepared on a Si substrate as described previously [19]. In brief, pieces of $\mathrm{Si}(100)$, approximately $15 \mathrm{~mm}$ x $15 \mathrm{~mm}$, were spin coated with photoresist (AZ4620 (Clariant)) then pyrolyzed at $1060{ }^{\circ} \mathrm{C}$ for $1 \mathrm{~h}$ in an atmosphere of $95 \% \mathrm{~N}_{2}$ and $5 \% \mathrm{H}_{2}$. After cooling to room temperature the PPF samples were removed from the furnace and 
stored under vacuum. The average surface roughness of samples was $\leq 0.6 \mathrm{~nm}$ and the surface resistivity was $18-25 \Omega /$ sq.

For CNT synthesis by thermal CVD, Al (5 nm) and Fe (5 nm) films were consecutively deposited on PPF samples using an Edwards 3000 e-beam evaporator. Stainless steel shadow masks were used to define disks of catalyst with diameters 2 $\mathrm{mm}$ and $100 \mu \mathrm{m}$. For the standard procedure, PPF samples were placed in a quartz tube (inner diameter $=28 \mathrm{~mm}$ ) and heated at $750{ }^{\circ} \mathrm{C}$ for $30 \mathrm{~min}$ under an atmosphere of $\mathrm{Ar}(1200 \mathrm{sccm})$ and $\mathrm{H}_{2}(800 \mathrm{sccm})$. Ethylene was introduced at $750{ }^{\circ} \mathrm{C}$ with gas flows of ethylene: $400 \mathrm{sccm}$, Ar: $300 \mathrm{sccm}$ and $\mathrm{H}_{2}: 200 \mathrm{sccm}$. Additionally, a small amount of water vapour was carried into the reaction chamber by bubbling 50-100 sccm Ar through water prior to feeding into the furnace. After the selected reaction time (20 -30 min), the chamber was cooled to below $100{ }^{\circ} \mathrm{C}$ under an Ar flow (2000 sccm) before removing the samples from the furnace.

For electrical and electrochemical measurements using VACNT arrays on PPF, a $\mathrm{Cu}$ strip was contacted with the PPF using silver paste, and the paste covered with epoxy. To insulate the VACNT array sidewalls, SU-8 2005 photoresist was spin coated over the array at $1000 \mathrm{rpm}$ for $1 \mathrm{~min}$. The sample was soft-baked on a hotplate (3 min at $65{ }^{\circ} \mathrm{C}$ followed by $5 \min$ at $95{ }^{\circ} \mathrm{C}$ ), followed by UV cross-linking (Karl Sauss Mask Aligner, $3.3 \mathrm{~mW} \mathrm{~cm}{ }^{-2}, 50 \mathrm{~s}$ exposure) and post-baking for $3 \mathrm{~min}$ at $65^{\circ} \mathrm{C}$ followed by $6 \mathrm{~min}$ at $95^{\circ} \mathrm{C}$. CNT tips were exposed by treating the sample with $\mathrm{O}_{2}$ plasma (0.1 Torr, $1 \mathrm{sccm}, 500 \mathrm{~W}, 6 \mathrm{~min})$. When VACNT samples were used as electrodes without sidewall insulation, PPF around the base of the VACNTs was covered with polymethyl methacrylate (PMMA, approximately $1 \mu \mathrm{m}$ ) and baked at 95 ${ }^{\circ} \mathrm{C}$ for $10 \mathrm{~min}$. The PMMA and exposed carbon substrate were protected with a shadow mask during $\mathrm{O}_{2}$ plasma treatment. 


\subsection{Instrumentation and measurements}

Scanning electron microscope (SEM), transmission electron microscope (TEM), high resolution TEM (HRTEM) and atomic force microscopy (AFM) images were obtained using Leica S 440, Hitachi H600, Jeol 2011 and Nanoscope III microscopes, respectively. For TEM characterization, CNTs samples were scraped off the substrate and suspended in ethanol with ultrasonication. A drop of the suspension was evaporated on a TEM holey carbon grid. For AFM measurements, the CNT suspension was dropped onto a freshly-cleaved mica sheet.

Two-probe conductance testing was carried out on an insulated, $\mathrm{O}_{2}$ plasmatreated, 2 mm-diameter VACNT pillar on PPF. A $100 \mathrm{~nm}$ layer of silver was sputtercoated on top of the assembly and a $\mathrm{Cu}$ wire was connected to the silver film using silver paste. A Cu strip was attached to the PPF as described above. The direct current $I-V$ response of the sample was obtained with a two-probe Hewlett Packard 4155A semiconductor parameter analyzer, by sweeping the bias voltage between -0.1 and 0.1 $\mathrm{V}$ at room temperature.

Raman characterisation of VACNTs was conducted using a Jobin Yvon LabRam Raman system. The incident HeNe laser was excited at $632.81 \mathrm{~nm}$; the spot width was approximately $700 \mathrm{~nm}$. As-grown VACNT samples on PPF were placed either vertically or horizontally on a movable platform, enabling the incident laser beam to focus on selected positions between the base and top of the VACNT pillar.

Electrochemical measurements were made with a $\mu$-Autolab III (ECO-Chemie, Utrect, The Netherlands) potentiostat. The VACNT-PPF structure functioned as the working electrode with a Pt wire auxiliary and saturated calomel electrode (SCE) reference. A glass cell with a hole in the bottom was clamped over the VACNTs; an O-ring defined a circular area of $3.4 \mathrm{~mm}$ in diameter that was exposed to the solution. 
Electrical connection between the VACNTs and the potentiostat was established through the $\mathrm{Cu}$ strip attached to the PPF substrate. Unless stated otherwise, cyclic voltammograms of hydroxymethyl ferrocene ( $1 \mathrm{mM}$ in $0.1 \mathrm{M}$ aqueous $\mathrm{KCl}$ solution) were obtained with scan rate $=100 \mathrm{mV} \mathrm{s}^{-1}$.

\section{Results and Discussion}

\subsection{VACNT synthesis and characterisation}

Vertically aligned CNTs were synthesized directly on PPF substrates. Fig. 1a shows that long (greater than $1 \mathrm{~mm}$ ) VACNT arrays with a bundle density greater than $10^{8}$ CNTs per $\mathrm{cm}^{2}$ (Fig. 1b) were achieved using the standard growth conditions. The CNT arrays appear to be very uniform with no obvious large-scale surface contamination (Fig. 1c). VACNTs were found to grow only where the catalyst layers were deposited: they could be grown over the entire PPF surface, or by use of a shadow mask to pattern the catalyst spots, in disks with diameters ranging from $2 \mathrm{~mm}$ to $100 \mu \mathrm{m}$ (Fig. 1d).
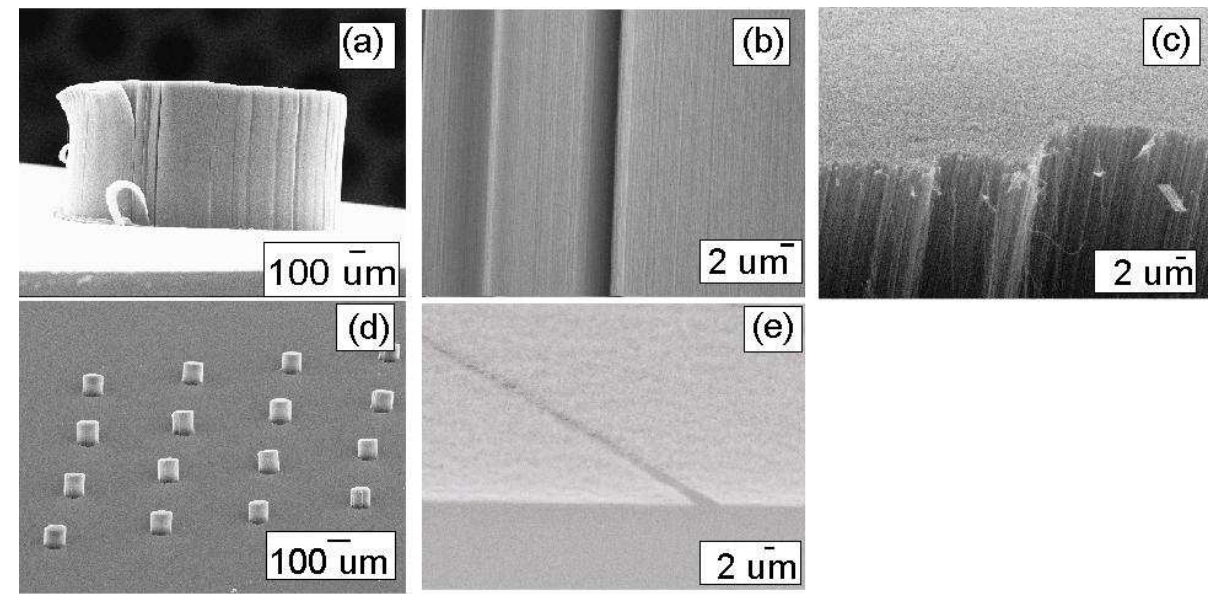

(e)

2 um

Fig. 1 - SEM images of (a) 2 mm diameter VACNT pillar synthesised on PPF; (b) VACNT sidewalls; (c) the top surface of a VACNT array; (d) $100 \mu \mathrm{m}$ diameter 
VACNT pillars on PPF and (e) CNTs synthesised on PPF using an Fe catalyst layer only.

The morphology of individual CNTs was investigated using AFM and TEM; representative images are shown in Figs. 2 and 3, respectively. Numerous AFM and TEM characterisations showed that the diameters of individual CNTs ranged from 10 to $20 \mathrm{~nm}$, consistent with the multiwalled (MW) structure revealed by HRTEM (Fig. 3b). All CNTs examined were found to have a small number of shells (less than 15) with large internal diameters. As demonstrated by the section of CNT shown in the HRTEM image of Fig. 3b, the outer shells of the MWCNTs are more defective than the inner shells, and kinked structures were frequently observed. The greater crystallinity of the inner shells can be attributed to the more limited accessibility of the inner shells to the etching gases (water vapour and hydrogen) during CNT synthesis.

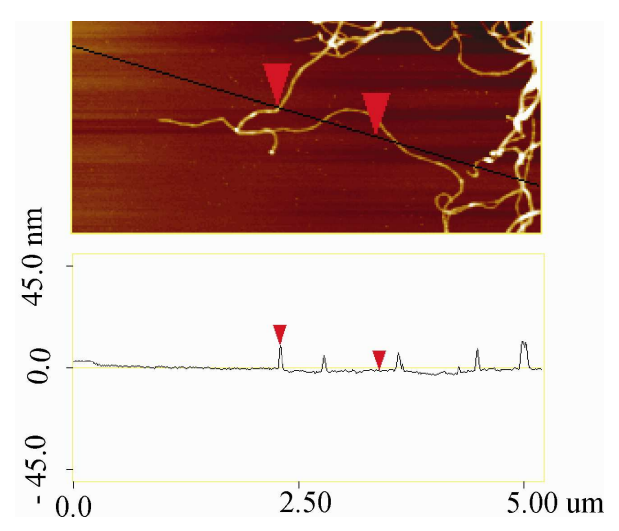

Fig. 2 - AFM topographical image and line profile of CNTs synthesized on PPF. 


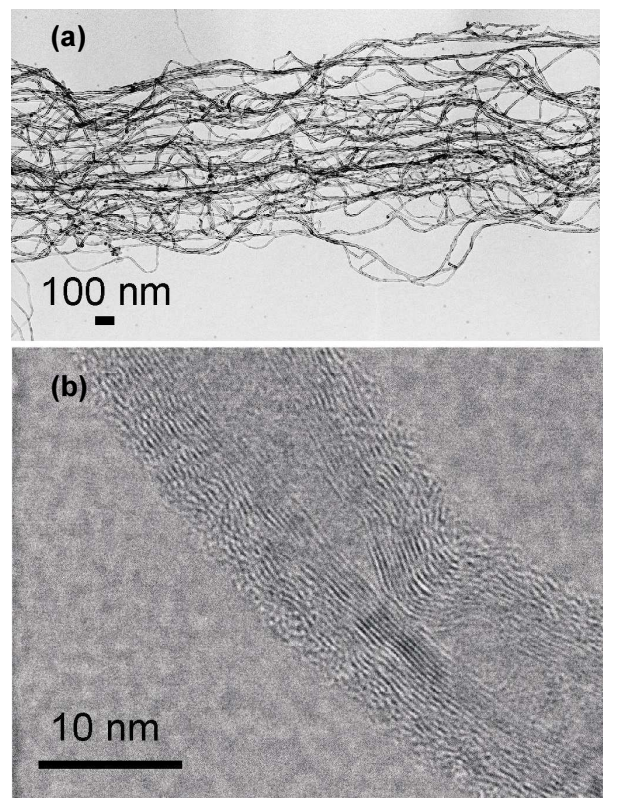

Fig. 3 - (a) Typical TEM image of CNTs synthesised on PPF substrates and (b) HRTEM image of a section of MWCNT.

Raman spectra were obtained of an as-grown VACNT pillar (2 mm diameter, $500 \mu \mathrm{m}$ long) on PPF. Fig. 4 shows spectra of the VACNT sidewalls, recorded at the base of the pillar and at $100 \mu \mathrm{m}$ intervals up the pillar (spectra (a) to (e)), and at the top surface of the pillar (spectrum (f)). The spectra show the expected D and G bands at 1330 and $1580 \mathrm{~cm}^{-1}$, respectively. For MWCNTs, the D band is attributed to the disturbance of the crystalline symmetry of the graphite lattice while the $G$ band is related to the graphitic lattice [20-23]. The large intensity of the D band indicates that there is a considerable amount of disorder-induced structure in the MWCNT samples. By comparing the $\mathrm{D}$ band/G band intensity ratio for spectra obtained at $100 \mu \mathrm{m}$ intervals along the VACNT sidewalls from the base of the pillar upwards (spectra (a) to (e)), it is evident that the contribution of disordered sidewall structures decreases 
from the base upwards. However, the Raman spectrum obtained from the top of the VACNT pillar (spectrum (f)) shows the largest $\mathrm{D}$ band/G band intensity ratio. This indicates, not surprisingly, that the open tips of the VACNT pillar contain a higher density of defects than the sidewalls.

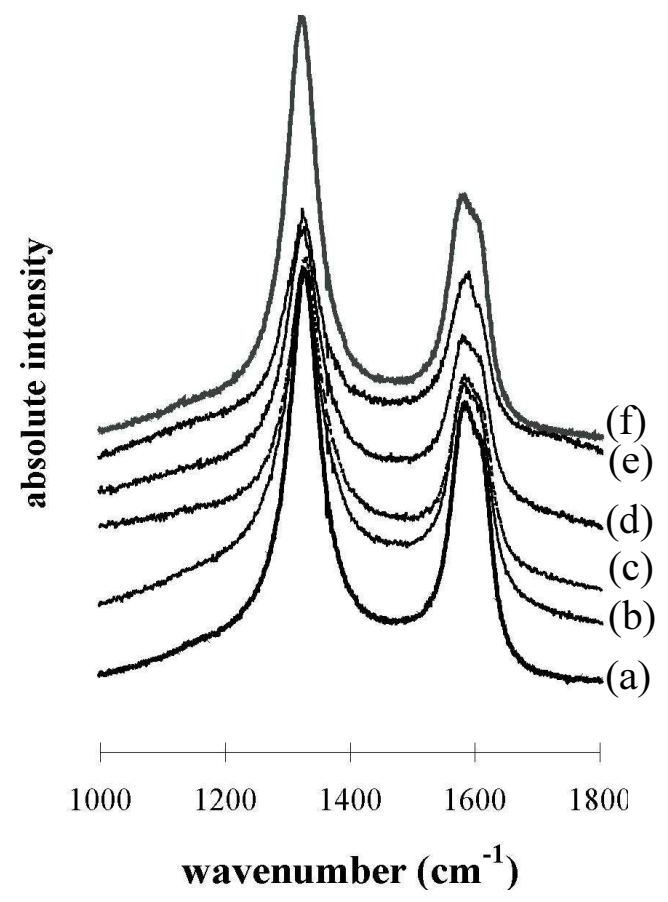

Fig. 4 - Raman spectra of a $500 \mu \mathrm{m}$-long, $2 \mathrm{~mm}$ diameter VACNT pillar on PPF. Spectra of the sidewalls were obtained at a distance of (a) 0 , (b) 100, (c) 200, (d) 300 and (e) $400 \mu \mathrm{m}$ from the base. Spectrum (f) was obtained from the top surface of the VACNT array.

For use of VACNTs as electrodes and for other electrical and electronic applications, the contact resistance between the VACNT array and the substrate must be low, and the array must adhere strongly to the conducting substrate. Using a 2point probe, the $I-V$ characteristic of an insulated, plasma-treated (see below) $2 \mathrm{~mm}$ VACNT pillar was obtained as described in the Experimental section. A linear and symmetric dependence of current on voltage was observed, confirming an Ohmic contact between the VACNTs and the PPF. The overall resistance of the assembly 
was $26.1 \Omega$, establishing the upper limit for the contact resistance. This low value indicates that the structures are suitable for use as electrodes. As-prepared, the VACNT arrays adhered strongly to PPF: when a $100 \mu$ m-diameter VACNT pillar was sheared off a PPF substrate by scraping with a piece of Si wafer, the Si support under the PPF layer was exposed, indicating that the adhesion of the VACNTs to the PPF was stronger than the adhesion of PPF to Si. Further, the attachment of VACNT arrays to PPF was stable to wetting and drying processes during use as electrodes (see below).

\subsection{Influence of synthesis conditions on growth of VACNTs}

The selection of a Fe/Al bilayer to catalyse CNT growth was based on the findings of other workers who report robust $\mathrm{CNT}$ growth on other substrates using Fe/Al catalyst systems and thermal CVD. Nakayama and co-workers reported growth of VACNTs on glass using Fe/Al bilayers [24], while Lui and Zhu (Wang) found that when using Fe(II) phthalocyanine as the catalyst, aligned CNTs grew better on Al-coated Si than on $\mathrm{Si}$ itself [12]. In a wide-ranging study of bilayer catalyst systems, $\mathrm{Ng}$ 's group found that $\mathrm{Al}$ was an excellent underlayer for all metal catalysts studied, including Fe [25]. In our work, use of a Fe catalyst layer, without pre-deposition of Al, led to the growth of short and randomly aligned CNTs on the carbon substrates (Fig. 1f). Although we were not able to directly establish whether aluminium is oxidised during catalyst preparation, the use of an $\mathrm{Ar}$ and $\mathrm{H}_{2}$ atmosphere during heating, and the observed Ohmic behaviour of the contact, strongly suggests that the catalyst layer remains metallic. We assume that the Al layers on PPF function as suggested for CVD growth on other substrates $[12,24]$, forming an alloy with Fe which increases the number of active Fe catalyst sites. Various combinations of $\mathrm{Al}$ and $\mathrm{Fe}$ thickness 
(ranging from 2 to $20 \mathrm{~nm}$ for each layer) were examined for their effect on VACNT growth. The fastest growth and best alignment of VACNTs was found for the 5 $\mathrm{nm} / 5 \mathrm{~nm}$ combination.

Fabrication of CNT arrays on graphitic carbon substrates raises the possibility that the substrate could act as a carbon source for CNT growth. To investigate this possibility, small spots of $\mathrm{Al} / \mathrm{Fe}$ catalyst layers were deposited on PPF and the sample was treated to the standard CVD conditions but without ethylene. No CNT growth was observed indicating that the substrate cannot act as the carbon source. The possibility that photoresist could be used as a carbon source was also investigated. AZ 4620 was spin-coated on top of $\mathrm{Al} / \mathrm{Fe}$ catalyst layers on PPF and the sample treated as above. Again, no CNT structures were found.

The effect of water vapour on the growth of VACNTs was monitored by varying the flow rate of the water vapour carrier gas (Ar) at constant ethylene flow rate for a growth time of $20 \mathrm{~min}$. Well-aligned CNTs were generated in the absence of water vapour, but longer CNTs were obtained in the presence of small amounts of water vapour. Fig. 5 shows that the length of VACNTs reached a maximum when the flow rate of the Ar carrier gas was 25\% that of ethylene (100 and $400 \mathrm{sccm}$ for Ar and ethylene, respectively). Hata and co-workers have demonstrated that water vapour has an accelerating effect on the growth rate of CNTs and they attribute the effect to etching of the carbon species which 'wrap' the catalyst particles [26]. Thus in the presence of water, the catalyst is maintained in a highly active state. However when the amount of water is too great, etching of the growing CNTs outweighs this effect reducing the net growth rate. It should be noted that in the present work, the lengths of VACNTs arrays were measured after 20 min CVD and it is possible that CNT growth terminated prior to that time. Hence the data of Fig. 5 do not allow growth rates to be 
determined, however the data exhibit a trend generally consistent with Hata's findings on the effect of water vapour on CNT growth rates.

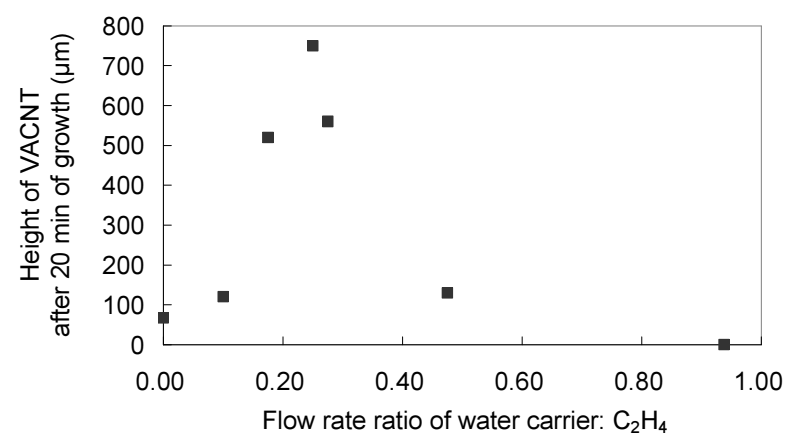

Fig. 5 - Effect of flow-rate ratio of Ar/water vapour : ethylene on the growth of VACNTs. Growth time was 20 min.

\subsection{Electrochemical characterisation}

The direct growth of CNTs on planar carbon substrates provides a simple method for fabricating CNT-based electrical and electrochemical devices. PPF has many attractive properties for such applications including good conductivity and physical robustness, low surface roughness [19], low cost and suitability for applications which require single use, disposable platforms. The characteristics of the VACNT-PPF assemblies as voltammetric electrodes were evaluated by obtaining cyclic voltammograms (CVs) of hydroxymethyl ferrocene $(\mathrm{FcOH}, 1 \mathrm{mM}$ in $0.1 \mathrm{M} \mathrm{KCl})$. Fig. 6a shows repetitive scan CVs recorded at an as-grown $120 \mu \mathrm{m}$ long VACNT pillar. The current density was calculated assuming that the electrode area corresponds to a disk with diameter $=2 \mathrm{~mm}$. Two closely-spaced oxidation peaks and a single reduction peak are evident on each scan. The peak currents decrease on repeat scans, reaching close to steady state after 10 scans. The overall appearance of the CVs suggests that diffusing redox probe is not a major contributor to the response and we assume that the response arises mainly from solution trapped within the VACNT array, giving typical thin-layer electrochemistry [27]. Hence the two oxidation peaks 
in the CVs of Fig. 6a are tentatively assigned to non-diffusing and diffusing FcOH at lower and higher potentials, respectively. The potential separation between the main oxidation and reduction peaks, $\Delta E_{\mathrm{p}}$, is clearly larger than the theoretical value of $0 \mathrm{~V}$ for a non-diffusing redox couple undergoing fast electron transfer [23]. The large $\Delta E_{\mathrm{p}}$ value indicates a relatively slow rate of electron transfer at the VACNT array and indicates that electron transfer at defect-free areas of CNT sidewalls dominates the response [4, 28-30].

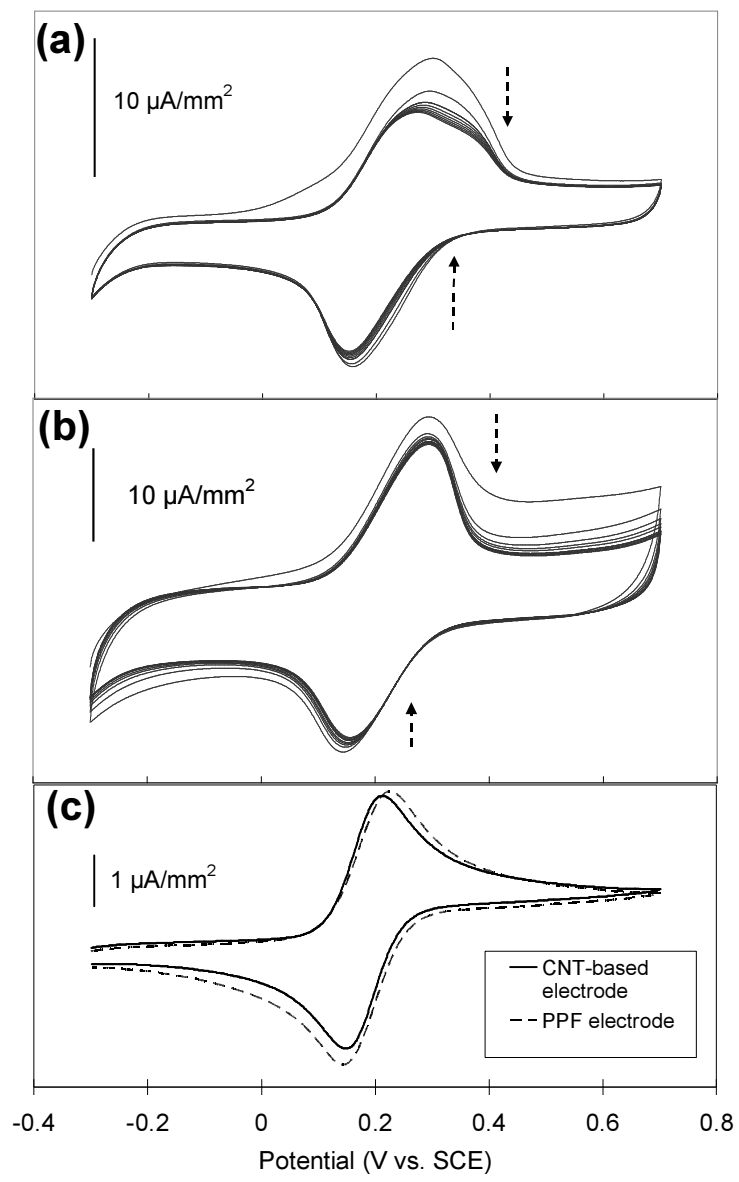

Fig. 6 - CV scans of $1 \mathrm{mM} \mathrm{FcOH}$ in $0.1 \mathrm{M} \mathrm{KCl}$ at a scan rate of $100 \mathrm{mV} \mathrm{s}^{-1}$ recorded at $2 \mathrm{~mm}$ diameter, $120 \mu \mathrm{m}$ long VACNT bundles on PPF. (a) as-grown VACNTs; (b) as-grown VACNTs after 6 min $O_{2}$ plasma treatment; (c) solid line: VACNTs insulated with SU-8 2005 followed by $O_{2}$ plasma treatment for 6 min; dashed line: bare PPF. Arrows indicate the direction of changes in current with 
repeat scans. Current densities were calculated assuming disk electrodes with diameter $=2 \mathrm{~mm}$ for VACNT electrodes and $3.4 \mathrm{~mm}$ for PPF.

The repeat $\mathrm{CV}$ scans of Fig. $6 \mathrm{~b}$ were obtained after $\mathrm{O}_{2}$ plasma treatment of the electrode in Fig. 6a, and show that both the peak and background currents are increased by plasma treatment. Plots of peak current $v s$ scan rate, and peak current $v s$ $(\text { scan rate })^{1 / 2}$ are shown in Fig. 7a. Neither plot is linear indicating that the response is not due to ideal thin-layer behaviour (which gives aa linear relationship between peak current and scan rate [27]) nor to ideal diffusion controlled behaviour (which gives a linear relationship between peak current and (scan rate) ${ }^{1 / 2}$ ) [31]. Considering the plots and the shape of the voltammograms of Fig. 7a, the response is attributed to thin-layer behaviour with slow electron transfer at the nanotube walls leading to $\Delta E_{\mathrm{p}}>0 \mathrm{~V}$ and giving the observed non-linear relationship between peak current and scan rate. A contribution from $i R$ drop may also contribute to the deviation from ideal thin-layer behaviour.
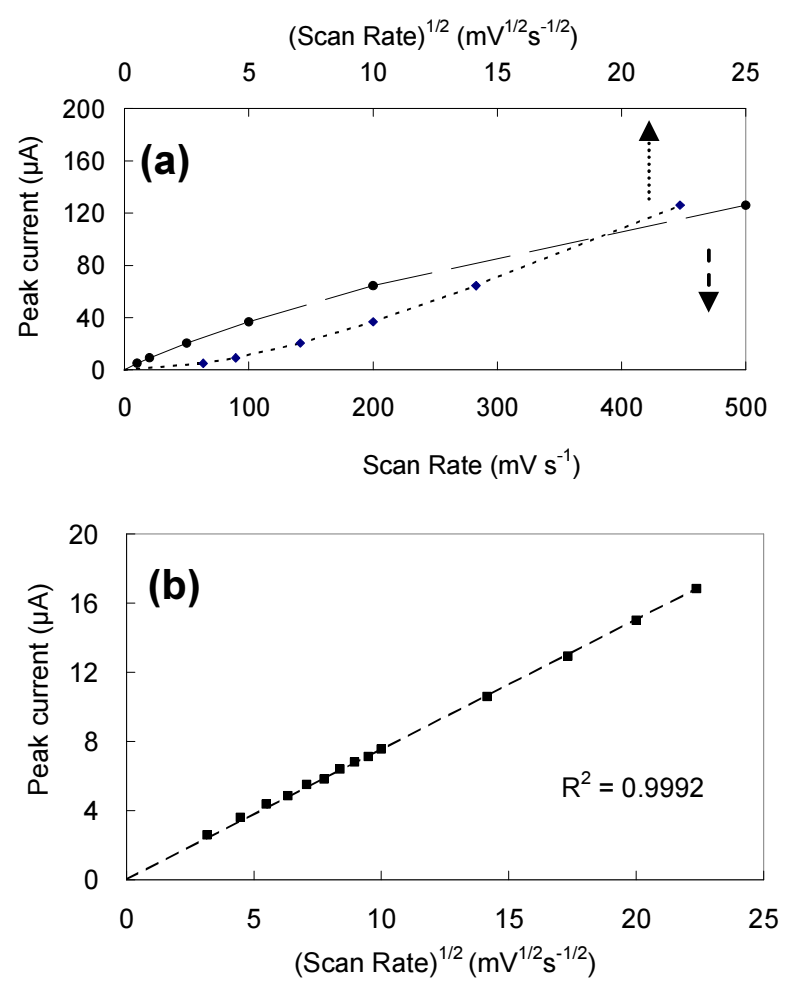
Fig. 7 - (a) Plots of CV peak current vs scan rate and peak current vs (scan rate) ${ }^{1 / 2}$ for the VACNT pillar electrode used to obtain the CVs in Fig. 6b, and (b) plot of CV peak current $v s$ (scan rate) ${ }^{1 / 2}$ for the VACNT pillar electrode used to obtain the CV in Fig. 6c (solid line). Voltammograms were recorded in $1 \mathrm{mM}$ FcOH in $0.1 \mathrm{M} \mathrm{KCl}$ solution.

Voltammograms obtained after insulation of the VACNT bundles with SU-8 2005 photoresist followed by $\mathrm{O}_{2}$ plasma treatment are shown in Fig. 6c (solid line). The CVs are consistent with a diffusion-controlled, fully reversible response [27]; this is confirmed by the linear relationship between peak current and (scan rate) $)^{1 / 2}$ (Fig. 7b) for $\mathrm{CVs}$ of $1 \mathrm{mM} \mathrm{FcOH}$ in $0.1 \mathrm{mM} \mathrm{KCl}$. A linear relationship between peak current and (scan rate) $)^{1 / 2}$ was also obtained when the experiment was repeated using 0.5 and $0.2 \mathrm{mM} \mathrm{FcOH}$ (data not shown). The $\Delta E_{\mathrm{p}}$ value of $59 \mathrm{mV}$ for the CVs of Fig. $6 \mathrm{c}$ indicates fast electron transfer between the VACNT electrode and $\mathrm{FcOH}^{+/ 0}$ [27] and demonstrates that the epoxy effectively covers the VACNT sidewalls, eliminating sites for slow electron transfer and leading to excellent electrochemical behaviour. The insulation step also prevents solution permeating into, and being trapped within, the VACNT bundle. $\mathrm{O}_{2}$ plasma treatment removes epoxy from the top of the VACNT array and exposes, and possibly generates additional, active sites at the VACNT tips, promoting fast electron transfer reactions. Electrodes prepared in this way are mechanically stable and robust.

Fig. 6c also shows a CV (dashed line) obtained at a bare PPF electrode. The current densities are very similar for the VACNT and PPF electrodes confirming that the VACNT electrodes are functioning as $2 \mathrm{~mm}$ diameter disks. In fact, determining the active area of the PPF electrode is difficult because of an unknown amount of 
'flattening' of the O-ring which defines the electrode area. Hence the very small difference in current density between the PPF and VACNT electrodes is attributed to uncertainty in the area of the PPF electrode.

Preparation of VACNT arrays on polished GC surfaces was also briefly examined using the same procedures as described for PPF. Preliminary CV experiments recorded using non-insulated VACNTs on GC were qualitatively similar to those described above for PPF. We did not apply SU-8 insulation to VACNT arrays on $\mathrm{GC}$, however it is expected that reversible, diffusion-controlled electrochemistry should be obtained at such surfaces, as observed for insulated arrays on PPF.

\section{Conclusion}

VACNT arrays can be successfully grown on planar graphitic carbon substrates using a bilayer Al/Fe catalyst and water-assisted thermal CVD. The arrays are uniform and highly aligned, and exhibit low contact resistance with PPF substrates. Direct growth of VACNT arrays on PPF, followed by insulation with epoxy and $\mathrm{O}_{2}$ plasma treatment, minimizes the number of manipulations required to prepare VACNT array electrodes. It is expected that the fabrication procedure will be suitable for VACNT preparation on other graphitic carbon substrates, such as glassy carbon and amorphous carbon films. In ongoing work we are preparing sub $10 \mu \mathrm{m}$ diameter bundles of VACNTs on PPF for use as microelectrodes.

\section{Acknowledgements}

The authors thank Richard D. Tilley and David Flynn (Victoria University of Wellington) and Neil Andrews (University of Canterbury) for assistance with electron microscopy, and Pablo Etchegoin (Victoria University of Wellington)' for assistance 
with Raman spectroscopy. The research is funded by the RSNZ Marsden Fund (contract UOC 0605), the MacDiarmid Institute for Advanced Materials and Nanotechnology and the University of Canterbury.

\section{REFERENCES}

[1] Choi WB, Bae E, Kang D, Chae S, Cheong BH, Ko JH, et al. Aligned carbon nanotubes for nanoelectronics. Nanotechnology 2004;15(10):S512-S6.

[2] Dai LM. Light-emitting polymers and carbon nanotube electron emitters for optoelectronic displays. Smart Mater Struct 2002;11(5):645-51.

[3] Li J, Stevens R, Delzeit L, Ng HT, Cassell A, Han J, et al. Electronic properties of multiwalled carbon nanotubes in an embedded vertical array. Appl Phys Lett 2002;81(5):910-2.

[4] Chou A, Bocking T, Singh NK, Gooding JJ. Demonstration of the importance of oxygenated species at the ends of carbon nanotubes for their favourable electrochemical properties. Chem Commun 2005(7):842-4.

[5] Gooding JJ, Chou A, Liu JQ, Losic D, Shapter JG, Hibbert DB. The effects of the lengths and orientations of single-walled carbon nanotubes on the electrochemistry of nanotube-modified electrodes. Electrochem Commun 2007;9(7):1677-83.

[6] Gooding JJ, Wibowo R, Liu JQ, Yang WR, Losic D, Orbons S, et al. Protein electrochemistry using aligned carbon nanotube arrays. J Am Chem Soc 2003;125(30):9006-7.

[7] Guiseppi-Elie A, Lei CH, Baughman RH. Direct electron transfer of glucose oxidase on carbon nanotubes. Nanotechnology 2002;13(5):559-64. 
[8] Sotiropoulou S, Chaniotakis NA. Carbon nanotube array-based biosensor. Anal Bioanal Chem 2003;375(1):103-5.

[9] Yu X, Chattopadhyay D, Galeska I, Papadimitrakopoulos F, Rusling JF. Peroxidase activity of enzymes bound to the ends of single-wall carbon nanotube forest electrodes. Electrochem Commun 2003;5(5):408-11.

[10] Gao Y, Liu J, Shi M, Elder SH, Virden JW. Dense arrays of well-aligned carbon nanotubes completely filled with single crystalline titanium carbide wires on titanium substrates. Appl Phys Lett 1999;74(24):3642-4.

[11] Agrawal S, Frederick MJ, Lupo F, Victor P, Nalamasu O, G. Ramanath. Directed growth and electrical- transport properties of carbon nanotube architectures on indium tin oxide films on silicon-based substrates. Adv Funct Mater 2005;15(12):1922-6.

[12] Wang BA, Liu XY, Liu HM, Wu DX, Wang HP, Jiang JM, et al. Controllable preparation of patterns of aligned carbon nanotubes on metals and metal-coated silicon substrates. J Mater Chem 2003;13(5):1124-6.

[13] Hiraoka T, Yamada T, Hata K, Futaba DN, Kurachi H, Uemura S, et al. Synthesis of single- and double-walled carbon nanotube forests on conducting metal foils. J Am Chem Soc 2006;128(41):13338-9.

[14] Talapatra S, Kar S, Pal SK, Vajtai R, Ci L, Victor P, et al. Direct growth of aligned carbon nanotubes on bulk metals. Nat Nanotech 2006;1(2):112-6.

[15] Parthangal PM, Cavicchi RE, Zachariah MR. A generic process of growing aligned carbon nanotube arrays on metals and metal alloys. Nanotechnology 2007;18(18). 
[16] Boskovic BO, Golovko VB, Cantoro M, Kleinsorge B, Chuang ATH, Ducati

C, et al. Low temperature synthesis of carbon nanofibres on carbon fibre matrices. Carbon 2005;43(13):2643-8.

[17] Smiljanic O, Dellero T, Serventi A, Lebrun G, Stansfield BL, Dodelet JP, et al. Growth of carbon nanotubes on ohmically heated carbon paper. Chem Phys Lett 2001;342(5-6):503-9.

[18] Tang H, Chen JH, Nie LH, Yao SZ, Kuang YF. Electrochemical oxidation of glutathione at well-aligned carbon nanotube array electrode. Electrochim Acta 2006;51(15):3046-51.

[19] Brooksby PA, Downard AJ. Electrochemical and atomic force microscopy study of carbon surface modification via diazonium reduction in aqueous and acetonitrile solutions. Langmuir 2004;20(12):5038-45.

[20] Ferrari AC, Robertson J. Interpretation of raman spectra of disordered and amorphous carbon. Phys Rev B 2000;61(20):14095.

[21] Li W, Zhang H, Wang C, Zhang Y, Xu L, Zhu K, et al. Raman characterization of aligned carbon nanotubes produced by thermal decomposition of hydrocarbon vapor. Appl Phys Lett 1997;70(20):2684-6.

[22] Liu K, Jiang K, Feng C, Chen Z, Fan S. A growth mark method for studying growth mechanism of carbon nanotube arrays. Carbon 2005;43(14):2850-6.

[23] Thomsen C, Reich S. Double resonant raman scattering in graphite. Phys Rev Lett 2000;85(24):5214 LP - 7.

[24] Nakayama Y, Pan LJ, Takeda G. Low-temperature growth of vertically aligned carbon nanotubes using binary catalysts. Jpn J Appl Phys 2006;45(1A):36971. 
[25] Ng HT, Chen B, Koehne JE, Cassell AM, Li J, Han J, et al. Growth of carbon nanotubes: A combinatorial method to study the effects of catalysts and underlayers. J Phys Chem B 2003;107(33):8484-9.

[26] Hata K, Futaba DN, Mizuno K, Namai T, Yumura M, Iijima S. Water-assisted highly efficient synthesis of impurity-free single-walled carbon nanotubes. Science 2004;306:1362-4.

[27] Bard AJ, Faulkner LR. Electrochemical methods: Fundamentals and applications. 2nd ed: John Wiley: New York; 2001: pp 455-458.

[28] Banks CE, Davies TJ, Wildgoose GG, Compton RG. Electrocatalysis at graphite and carbon nanotube modified electrodes: Edge-plane sites and tube ends are the reactive sites. Chem Commun 2005(7):829-41.

[29] Banks CE, Moore RR, Davies TJ, Compton RG. Investigation of modified basal plane pyrolytic graphite electrodes: Definitive evidence for the electrocatalytic properties of the ends of carbon nanotubes. Chem Commun 2004(16):1804-5.

[30] Moore RR, Banks CE, Compton RG. Basal plane pyrolytic graphite modified electrodes: Comparison of carbon nanotubes and graphite powder as electrocatalysts. Anal Chem 2004;76(10):2677-82.

[31] Reference 27, pp $228-232$. 
Figure captions

Fig. 1 - SEM images of (a) 2 mm diameter VACNT pillar synthesised on PPF; (b) VACNT sidewalls; (c) the top surface of a VACNT array; (d) $100 \mu \mathrm{m}$ diameter VACNT pillars on PPF and (e) CNTs synthesised on PPF using an Fe catalyst layer only.

Fig. 2 - AFM topographical image and line profile of CNTs synthesized on PPF.

Fig. 3 - (a) Typical TEM image of CNTs synthesised on PPF substrates and (b) HRTEM image of a section of MWCNT.

Fig. 4 - Raman spectra of a $500 \mu \mathrm{m}$-long, $2 \mathrm{~mm}$ diameter VACNT pillar on PPF. Spectra of the sidewalls were obtained at a distance of (a) 0, (b) 100, (c) 200, (d) 300 and (e) $400 \mu \mathrm{m}$ from the base. Spectrum (f) was obtained from the top surface of the VACNT array.

Fig. 5 - Effect of flow-rate ratio of Ar/water vapour : ethylene on the growth of VACNTs. Growth time was 20 min.

Fig. 6 - CV scans of $1 \mathrm{mM} \mathrm{FcOH}$ in $0.1 \mathrm{M} \mathrm{KCl}$ at a scan rate of $100 \mathrm{mV} \mathrm{s}$ recorded at $2 \mathrm{~mm}$ diameter, $120 \mu \mathrm{m}$ long VACNT bundles on PPF. (a) as-grown VACNTs; (b) as-grown VACNTs after 6 min $O_{2}$ plasma treatment; (c) solid line: VACNTs insulated with SU-8 2005 followed by $O_{2}$ plasma treatment for 6 min; dashed line: bare PPF. Arrows indicate the direction of changes in current with 
repeat scans. Current densities were calculated assuming disk electrodes with diameter $=2 \mathrm{~mm}$ for VACNT electrodes and $3.4 \mathrm{~mm}$ for PPF.

Fig. 7 - (a) Plots of CV peak current vs scan rate and peak current vs (scan rate $)^{1 / 2}$ for the VACNT pillar electrode used to obtain the CVs in Fig. 6b, and (b) plot of CV peak current vs (scan rate) ${ }^{1 / 2}$ for the VACNT pillar electrode used to obtain the CV in Fig. 6c (solid line). Voltammograms were recorded in $1 \mathrm{mM}$ FcOH in 0.1 M KCl solution. 